\title{
Molecular Docking of New Active Compounds Towards the Acetylcholinesterase Enzyme
}

\author{
${ }^{1}$ Lemaoui Tarek, ${ }^{1}$ Hammoudi Nour El Houda, ${ }^{1}$ Benguerba Yacine and ${ }^{2}$ Attoui Ayoub \\ ${ }^{1}$ Laboratoire des Matériaux Polymères Multiphasiques, LMPMP, Université Ferhat ABBAS Sétif-1, 19000 Sétif, Algeria \\ ${ }^{2}$ Laboratoire de Biochimie Appliquée, Université Ferhat ABBAS Sétif-1, 19000 Sétif, Algeria
}

\author{
Article history \\ Received: 25-12-2019 \\ Revised: 11-02-2020 \\ Accepted: 29-02-2020 \\ Corresponding Author: \\ Lemaoui Tarek \\ Laboratoire des Matériaux \\ Polymères Multiphasiques, \\ LMPMP, Université Ferhat \\ ABBAS Sétif-1, 19000 Sétif, \\ Algeria \\ Email: tareklemaoui19@gmail.com
}

\section{Introduction}

Molecular docking is a computational process for the insertion of ligands (often small molecules) into the binding site of their receptor (macromolecular target). Docking algorithms and scoring functions can generate structures of receptor-ligand complexes, ranking compounds and estimating binding energies/affinities (Sousa et al., 2013). The objective of this work is to perform a molecular docking in order to determine the affinity of a total of DL0410 and its 49 DL0410 derivatives and four marketed molecules, tacrine, galantamine, donepezil and rivastigmine). Which were taken from the literature (Pang et al., 2017).

\section{Molecular Docking Study}

\section{Protein Preparation, Grid Generation and Ligand Preparation}

Protein enhancement and grid generation are required steps for suitable docking. The threedimensional X-ray crystallographic structure of the acetylcholinesterase protein was taken from protein data bank. Protein was prepared by eliminating water molecules, needless atoms, removing the alternate conformations and adding hydrogen.

The grid generation describes a region in a receptor where binding interaction can happen. Before launching the molecular docking, the geometry of all molecules needs to be optimized (Vora et al., 2019).
All these steps were performed using (Schrodinger, Maestro) software.

\section{Results and Discussion}

Molecular docking study was performed for 56 Acetylcholinesterase Inhibitors compounds. The molecule 3.2 has the highest score $(-13,681)$, the different types of interactions which may show the binding of the molecule 3.2 with the AChE are shown in (Fig. 1). The compound 3.2 was situated in the active site, interacting with four residues, the nitrogen 8403 interacts with TRP 279 by Pi -cation interaction, the aromatic structure of compound adopted a suitable orientation for its binding to PHE330 via Pi-cation interaction, carbonyl, Hydrogen 8451 forming hydrogen bonds with PHE288, SER122, respectively.

\section{Compound 3.4.}

The Nitrogen (8379) interacts with the Amino acid TRP279 assuring a pi-cation interaction, (PHE288, SER122) forming hydrogen bonds with (carbonyl 8387, Hydrogen 8460) respectively (Fig. 2).

\section{Compound 2.3}

Presence of hydrogen bond between the ARG289 and carbonyl8400, Pi-Pi staking between the aromatic ring of the compound and TRP 279, pi-cation between N8383 and TRP84. Three-dimensional docking model of compound 2.3 with AChE is shown in Fig. 3. 


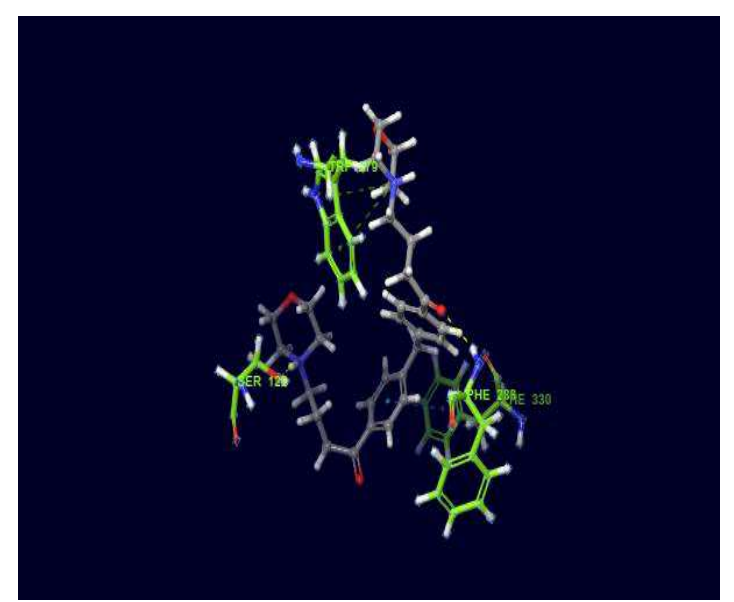

Fig. 1: Three-dimensional docking model of compound $\mathbf{3 . 2}$ with AChE

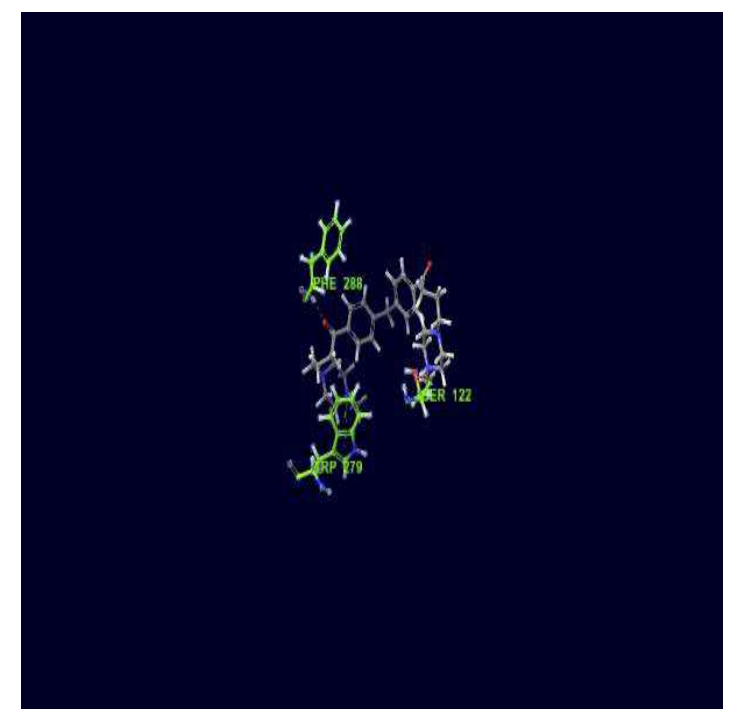

Fig. 2: Three-dimensional docking model of compound 3.4 with AChE

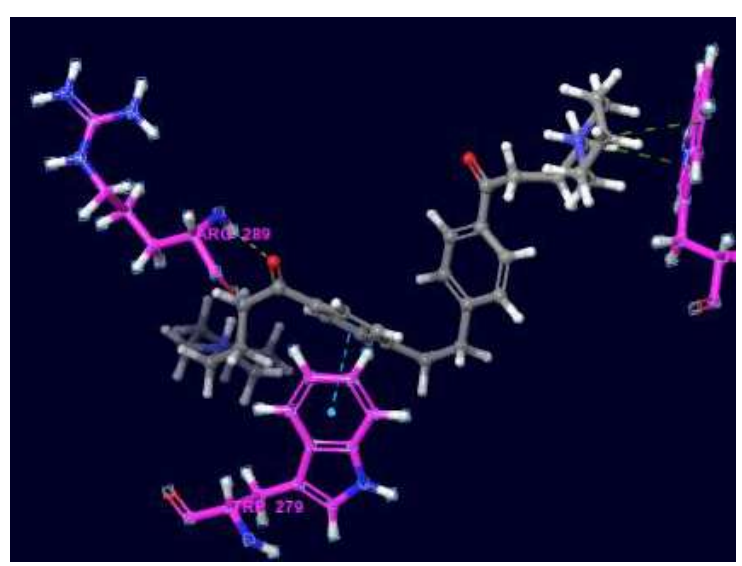

Fig. 3: Three-dimensional docking model of compound $\mathbf{2 . 3}$ with $\mathrm{AChE}$

\section{Compound 1.18}

The aromatic ring of the compound interacts with TRP279 showing a pi-pi staking interaction, Nitrogen 8412 forming hydrogen bonds interaction with PHE330 and TRP 84, carbonyl8404 forms a hydrogen bond with PHE288, in addition to this interaction, there is another interaction between N8408 and TYR334 called pi-cation interaction. Three-dimensional docking model of compound 2.3 with AChE is shown in Fig. 3.

\section{Donepezil (Marketed Molecule)}

The nitrogen 8394 interacts with ASP72 by a salt bridg interaction. The aromatic structure of compound supposed a good orientation for its binding to 334TYR via Pi-cation interaction, carbonyl 8389 forms a hydrogen bonds with PHE288 and the aromatic ring interact with TRP279 by pi-pi staking interaction.

Table 1: Docking score of the investigated compounds

\begin{tabular}{ll}
\hline Compound & Docking score \\
\hline 3.2 & $-13,681$ \\
3.4 & $-13,549$ \\
2.3 & $-13,521$ \\
1.18 & $-13,421$ \\
Donepezil & $-12,978$ \\
2.2 & $-12,902$ \\
6.2 & $-12,83$ \\
3.5 & $-12,353$ \\
2.1 & $-12,325$ \\
Galanthamine & $-12,11$ \\
5.3 & $-12,1$ \\
6.4 & $-11,896$ \\
2.1 & $-11,29$ \\
7.7 & $-10,898$ \\
6.1 & $-10,633$ \\
1.15 & $-10,55$ \\
3.3 & $-10,531$ \\
1.7 & $-10,29$ \\
4.5 & $-10,151$ \\
2.6 & $-10,122$ \\
Rivastigmine & $-10,013$ \\
2.7 & $-9,711$ \\
1.17 & $-9,535$ \\
1.14 & $-9,213$ \\
3.5 & $-8,716$ \\
3.1 & $-8,515$ \\
6.1 & $-8,191$ \\
5.2 & $-7,717$ \\
2.9 & $-7,629$ \\
4.4 & $-7,494$ \\
2.4 & $-6,509$ \\
2.8 & $-6,349$ \\
7.4 & $-6,213$ \\
4.3 & $-5,924$ \\
4.1 & $-5,918$ \\
5.1 & $-5,207$ \\
4.2 & $-5,189$ \\
7.3 & $-4,905$ \\
2.9 & $-4,842$ \\
& $-2,875$ \\
\hline & \\
\hline &
\end{tabular}




\section{Docking Score}

Table 1 represents the docking scores results of the investigated molecules; the scores are arranged in descending order from smallest the negative value to the major $t$ negative value. It was found that ten molecules have no poses in the receptor site of $\mathrm{AChE}$ and four molecules with better scores when compared to the score of the marketed molecule (Donepezil).

\section{Conclusion}

In this work a docking study was carried out on 56 acetylcholinesterase inhibitory molecules, the results obtained indicate that most of these molecules have a very good affinity towards the acetylcholinesterase enzyme.

\section{Acknowledgment}

The authors gratefully acknowledge the General Directorate of Scientific Research and Technological Development.

\section{Author's Contributions}

Lemaoui Tarek: Work realization (calculation and redaction).

Hammoudi Nour El Houda: Provide ideas and some proposals.

Attoui Ayoub: Orientation and correction of the biological part.

Benguerba Yacine: Orientation and correction of the molecular modeling part.

\section{Ethics}

This research was subjected to ethical clearance from the faculty of technology, Ferhat ABBAS university setif-1, Setif/Algeria.

\section{References}

Morris, G.M. and M. Lim-Wilby, 2008. Molecular Docking. In: Molecular Modeling of Proteins, Kukol, A. (Ed.), Humana Press, pp: 365-382.

Pang, X., H. Fu, S. Yang, L. Wang and A.L. Liu et al., 2017. Evaluation of novel dual acetyl-and Butyrylcholinesterase inhibitors as potential antiAlzheimer's disease agents using pharmacophore, 3D-QSAR and moleculardocking approaches. Molecules, 22: E1254-E1254. DOI: $10.3390 /$ molecules22081254

Sousa, S.F., A.J. Ribeiro, J.T.S. Coimbra, R.P.P. Neves and S.A. Martins et al., 2013. Proteinligand docking in the new millennium-a retrospective of 10 years in the field. Curr. Medicinal Chem., 20: 2296-2314.

DOI: 10.1002/jmr.2471

Vora, J., S. Patel, S. Sinha, S. Sharma and A. Srivastava et al., 2019. Molecular docking, QSAR and ADMET based mining of natural compounds against prime targets of HIV. J. Biomolecular Structure Dynam., 37: 131-146. DOI: $10.1080 / 07391102.2017 .1420489$ 